\title{
Optimizing the Passenger Throughput at an Airport Security Checkpoint Xie Youyou
}

\author{
Jilin University, Changchun China \\ e-mail:xieyy650@163.com
}

Keywords: Queuing theory; Jackson network; CA model.

\begin{abstract}
The tension between desires to maximize security while minimizing inconvenience to passengers at the airport security checkpoint has increased during few year time. The purpose of this paper is to construct a model to detect the bottlenecks hidden in the current system hence ease the tension by improving our model.

First we focus on simulating the system. We divide the process into two main sections and take them into consideration separately. We then build two models in regard to their characteristics: the first one is based on the queuing theory, while the second one is based on the Jackson network theorem considering the second section does not fit the condition of reversible Markov chain process.

Afterwards, we analyze the given data in the model. It is followed by the hidden bottlenecks in the system exposing themselves. Noticing the bottlenecks, we aim at optimizing and improving the current model to reduce the waiting time in the next step. The time varying model has provided us an insight into allocating the resources in accordance to time. Moreover, we reduce the variance of waiting time by 90 percent by applying the control varieties method.

Next, we focus on analyzing how social norms impact our model using the cellular automaton model. Then we make some modification to optimize the throughput and minimize the variance. Last, we give some recommendation to the manager based on the results of our model.

To sum up, our model is an integral and adaptable model that is suitable for further assessment.
\end{abstract}

\section{Introduction}

1.1 Background. Passing through the security checkpoints with high efficiency has always been an inevitable task for the passengers. However, due to the rapid growth in the number of passengers and carry-on baggage(mainly electronic devices),the financial constraints, as well as the lack of human resources, more and more complaints and public critics has urged the administer of the airport to improve the efficiency of the security check given the high priory of the security and safety factor. Accordingly, we are supposed to set up a model to maximize the security while minimize the wasted time.

1.2 Current solutions. Such a process has been adopted by the U.S. Transportation Security Agency (TSA) in order to reduce the current pressure of security check. The checkpoint is divided into four zones: Zone $\mathrm{A}$ is for document check, Zone $\mathrm{B}$ is for baggage and body screening, Zone $\mathrm{C}$ works as item collect area and exit, and Zone $\mathrm{D}$ as the additional screening when passengers fail the inspection in Zone B.

We will base some parts of our model design on the Pre-Check system. In the meantime, we will use the pre-exist Pre-Check system as a basic comparison for our further modified models.

\subsection{Our Approaches.}

First we clarify our goals. The model that we build should:

- simulate how the system works.

-be clear in every step.

-be adaptive and flexible.

1.4 Notation. First we make the following notation. 


\begin{tabular}{ll}
\hline Symbol & Meaning \\
$\lambda$ & number of TSA officers in ID checking section \\
$\mu$ & mean number of arrivals per minute \\
$\rho$ & mean number of people checked per minute \\
$L_{s}$ & probability of having to wait in queue \\
$L_{q}$ & the average number of passengers in the system \\
$W_{s}$ & the average number of passengers in the the queue \\
$W_{q}$ & the average time of per passenger spends waiting in the system \\
\hline
\end{tabular}

\section{Simulating Checking Process}

We have noticed that for the majority of the passengers, the most time-consuming process is the ID check and bag-screening section. Therefore, we take Zone A and Zone B into consideration exclusively.

2.1 Simulation on Zone A via Queuing theory. Queuing theory is a well researched and widely used quantitative analysis technique. In this section, we will introduce the concept of the basic queuing theory and demonstrate of applicability to our description on the flow in Zone A.

2.1.1 Assumptions.

We then make assumptions as follows.

- Arrivals are checked on a $F I F O^{1}$ basis.

- People arriving per minute $\lambda$ and document checked per minute $\mu$ is constant.

- There is no cutting in line in the queue.

-Every lane is independent to others.

2.1.2 Analysis.

-We first consider passengers with Pre-Check arriving at their exclusive check table in Zone A. According to the given data, here $\lambda=6.667$. When TSA officer 1 takes in charge of the process, $\mu_{1}=6.000$, while TSA officer 2 takes in charge, $\mu_{2}=4.615$. The average $\mu=5.308$. We can see from here, $\mu \approx \lambda$, which means the chance of having to wait is rather small. Comparing with the single-channel queuing model, we can find that the problem arises because the basic model assumes that $\mu, \lambda$ on average. Therefore, the traditional single-channel queuing system can not be apply in this case.

- Then we consider passengers arriving at regular check table in Zone A. Assuming that there will be more than one TSA officer for the check, we try applying multiple-channel queuing model in this case.

We assume that:

-checking times at each channel are described by a negative exponential probability distribution with the rate $\mu_{i}$.

-arrivals will join the first available line.

Other assumptions listed in the single-channel model may apply.

According to the given data, $\mu_{1}=6: 000, \mu_{2}=4: 615$ with the equation 


$$
W_{q}=\frac{\rho(s \rho)^{k}}{s !(1-\rho)^{2}}\left[\sum_{k=0}^{s-1} \frac{(s p)^{k}}{k !}+\frac{(s p)^{s}}{s !(1-\rho)}\right]^{-1}
$$

we can have results of $L_{S}, L_{q}, W_{s}, W_{q}$.

Table 1: When $\mathrm{s}=2$

\begin{tabular}{lllll}
\hline$\mu$ & $L_{s}$ & $L_{q}$ & $W_{s}$ & $W_{q}$ \\
\hline$\mu_{1}$ & 0.840 & 0.455 & 10.909 & 0.909 \\
$\mu_{2}$ & 1.121 & 0.621 & 14.561 & 1.574
\end{tabular}

We can see from Table 1, there is limited chance that people in regular lanes have to wait for document check, even though there only are two lanes open. Therefore, we come to the conclusion that people do not have much time wasted in Zone A.

However, it is not often the case in actual world. That is because the given data, in which $\mu \approx \lambda$ may be collected during the slack hours, when the passenger volume is small.

Though the basic queuing theory provides a vision on the security check simulation, it is not very adaptive and flexible for each step. In short, we need to modify the basic model for simulation on Zone A. This will be further discussed in the next section.

\subsection{Simulation on Zone B via Jackson Network}

After leaving Zone A, passengers proceed to the screening section in Zone B.

In this section, we assume the time interval between two passengers leaving Zone $\mathrm{A}$ is described by a certain distribution.

There are two possible circumstances:

1. There is a second passenger coming for the ID check after the first one leaves, with the probability $\mathrm{p}$. In this case, the time interval between the two passengers D1 equals to the checking time for the second passenger

$$
D_{1}=\frac{\mu}{x+\mu}
$$

2. There is no passengers in the system after the first one leaves. That is, the second passenger will arrive after the first one leaves. In this case, the time interval between two passengersD2 equals to the time gap and the checking time for the second passenger.

$$
\begin{aligned}
& D_{2}=\frac{\lambda}{x+\lambda}+\frac{\mu}{x+\mu} \\
& D(x)=p D_{1}+(1-p) D_{2}=\frac{\lambda}{x+\lambda}
\end{aligned}
$$

With a Laplace transform from $\mathrm{D}(\mathrm{x})$ to $\mathrm{d}(\mathrm{t})$, we have:

$$
d(t)=\lambda e^{-\lambda t}
$$

Therefore, the output is described by a Poisson distribution with $\lambda$.

\subsubsection{Jackson Network}

Jackson network is a class of queueing network where the equilibrium distribution is particularly simple to compute as the network has a product-form solution.

\subsubsection{Assumptions}

First, we make some key assumptions as follows.

- There are three regular lanes and one Pre-check lane in Zone B.

-Pre-check Passengers will join the Pre-check lane, despite there might be shorter queue in regular lanes.

- Regular passengers can choose either of the regular lanes. 
-Queues in each node remain the same length.

In Figure 1 we describe the flow used in the model. We first divide the security check process in Zone $\mathrm{B}$ into 3 parts.

1. Passengers choose one lane to wait for the security check. The chance is equal.

2. Passengers choose either millimeter wave body scanner or metal detector. The chance is equal.

3. Passengers either leave the checkpoint or enter Zone D.

Then we set each section which may form a queue as a node. Through observation into the actual case, for the process from node 1 to node 2 ,

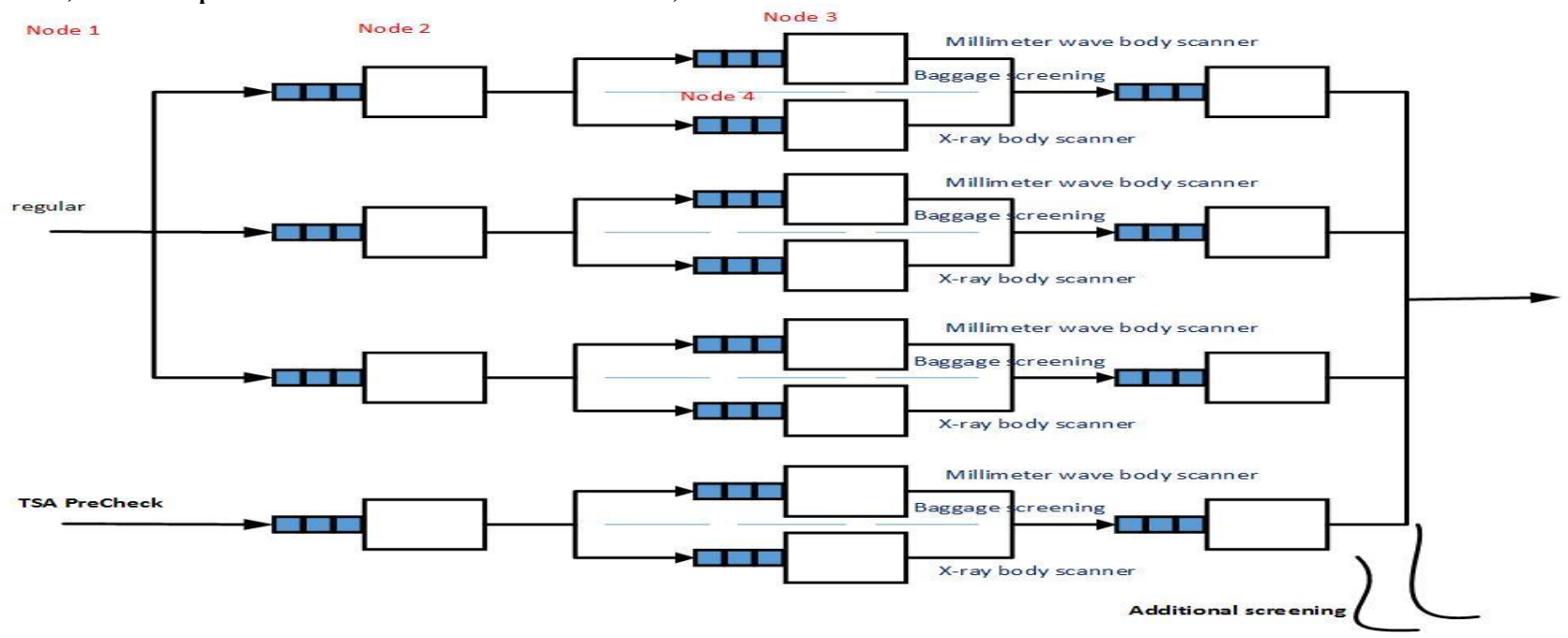

Figure 1: Simulation on Zone B

-each node can be considered into a multi-channel queuing system with average checking time $\mu_{i}$

-as proven above, the input of each node can be described by a Poisson distribution with $\lambda_{i}$;

-passengers will arrive at the next node $\mathrm{j}$ from node $\mathrm{i}$ with the probability $\mathrm{p}_{i j}$, or leave the system with the probability $1-\sum_{j=1}^{N} p_{i j}$

We have the overall arrival rate to node $i$,

$\Lambda_{i}=\lambda_{i}+\sum_{j=1}^{N} \Lambda_{j}, i=1,2, \cdots, N$

It fits the definition of the open Jackson network.

Using the equation

$$
P\left(Y_{i}=n\right)=p\left(Y_{i}=0\right) \frac{\lambda_{i}^{n}}{\prod_{j=1}^{n} \mu_{i}(j)}
$$

$P\left(Y_{i}=0\right)=\left(1+\sum_{n=1}^{\infty} \frac{\lambda_{i}^{n}}{\prod_{j=1}^{n} \mu_{i}(j)}\right)^{-1}$

, with $N=2, \lambda_{1}=4: 615$, we can get the x such that the probability $\pi(x)=\prod_{j=1}^{n} \mu_{i}(j)$ achieves its maximum value. Next, we let $x=L q$. Besides, the model can apply to the Pre-check section as well. Then we have the results shown in the table 2 below. Hence, we have the results shown below. On the other hand, for the process from node 2 to node 3 and node 4 , we observe that there is no 
arrivals from outside and no departures from the network, which fits the definition of the closed Jackson network. As a result, we can apply the Jackson network into the actual case and determine the stationary probabilities.

Table 2: Closed Jackson Network Result for Regular Lanes

\begin{tabular}{llllll}
\hline node & Model & $L_{s}$ & $L_{q}$ & $W_{s}$ & $W_{q}$ \\
\hline 1 & $\mathrm{M} / \mathrm{M} / 3$ & 0.123 & 0 & 0.080 & 0.030 \\
2 & $\mathrm{M} / \mathrm{M} / 1$ & 1.123 & 1.000 & 1.825 & 1.625 \\
3 & $\mathrm{M} / \mathrm{M} / 1$ & 0.123 & 0 & 0.133 & 0 \\
\hline
\end{tabular}

\subsubsection{Analysis}

After analyzing the above data, we get the following results:

Table 3: Closed Jackson Network Result for Pre-check lane

\begin{tabular}{llllll}
\hline node & Model & $L_{s}$ & $L_{q}$ & $W_{s}$ & $W_{q}$ \\
\hline 1 & $\mathrm{M} / \mathrm{M} / 1$ & 0.334 & 0 & 0.005 & 0 \\
2 & $\mathrm{M} / \mathrm{M} / 1$ & 1.334 & 1.000 & 0.800 & 0.600 \\
3 & $\mathrm{M} / \mathrm{M} / 1$ & 0.334 & 0 & 0.067 & 0
\end{tabular}

- For the process from node 1 to node 2 , the most time-consuming process is in node 1 , which refers to the waiting area in Zone B.

-For the process from node 2 to node 3 and node 4 , the most time-consuming process is in node 3, which refers to the millimeter body scanner section in Zone B.

-Despite the results have shown the tendency of the status, the size of the queue is rather small. We then suppose that the data is collected during the slack hours.

- The advantage of the bigger $\mu$ for pre-check lane is not reflected in the result. We will modify the model in regard to the bottlenecks we have found.

\section{Improvements upon the Simulation}

\subsection{Optimizing Allocation for Time-Varying model}

During the simulation in Zone A, we came to a conclusion that little time is spent in the document checking section. However, as is often the case, we not only suffer from inconvenience when few lines are open at the peak time, but also feel uneasy when staff outnumber passengers in slack hours. Therefore, it is necessary to take variance of time into consideration. In this part, we aim at making better use of the resources in reaction to the passenger volume of the time in Zone A. We have simulated a time-varying model and make a graph revealing the relation among passenger volume, flight volume and time in a day.

\subsubsection{Model Modifying}

In the first section, we have derived the equation

$$
\rho=\frac{\lambda}{\mu}
$$

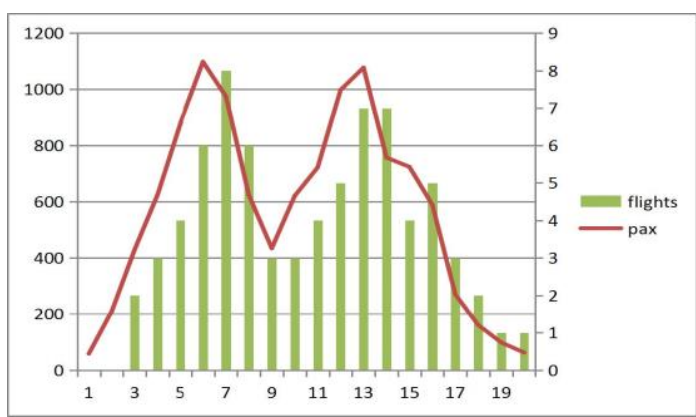

Figure 2: Passenger Volume and Flight Volume Changes in accordance to time in a Day 


$$
W_{q}=\frac{\rho(s \rho)^{k}}{s !(1-\rho)^{2}}\left[\sum_{k=0}^{s-1} \frac{(s \rho)^{k}}{k !}+\frac{(s \rho)^{s}}{s !(1-\rho)}\right]^{-1}
$$

and results of $L_{S}, L_{q}, W_{s}, W_{q}$ based on it.

Second, we further derive

$$
L_{q}(s)=\frac{(\rho)^{s+1}}{(s-\rho) \sum_{k=0}^{s-1} \frac{(\rho)^{k}}{(s-1) ! \frac{(s-k)}{k !}}}
$$

Considering the relation between ${ }^{2}$ and s, we simulate s=2, 3, 6 .

Third, we give some restriction regarding to the actual cases.

- $L q<l$, where $l$ denotes the maximal length of the queue. According to the restriction given by IATA, we assume that $l=12$.

$$
\text { - } P_{\min } \leq C(\rho, s) \leq P_{\max }
$$

We set our goal function as the maximum of efficiency shown below

$$
E_{\max }=p_{i} C(\rho, s) k \mu+q_{w}\left(l-L_{q}\right)-c s
$$

, where $\mathrm{c}$ denotes costs we spend for opening every lane per minute. Therefore, we can have the optimized model for s by find the solution of the goal function.

However, it is difficult to solve it by hands. Thus, we use marginal analysis to find the solution. When the goal function reaches its maximum, s_ will satisfy the inequalities below.

$$
\begin{aligned}
& \frac{c_{s}}{\mu p_{s}} \leq \frac{q_{w}}{p_{s}} \rho\left(W_{q}\left(s_{*}-1\right)-W_{q}\left(s_{*}\right)\right)-s_{*}\left(C\left(\rho, s_{*}-1\right)-C\left(\rho, s_{*}\right)\right]+C\left(\rho, s_{*}-1\right) \\
& \frac{c_{s}}{\mu p_{s}} \geq \frac{q_{w}}{p_{s}} \rho\left(W_{q}\left(s_{*}\right)-W_{q}\left(s_{*}\right)+1\right)-s_{*}\left(C\left(\rho, s_{*}\right)-C\left(\rho, s_{*}+1\right)\right]+C\left(\rho, s_{*}+1\right)
\end{aligned}
$$

If the $S_{*}$ we get satisfies the restriction mentioned above, then we have the optimized s. If not, let $\mathrm{s}=$ $\mathrm{s}+1$, and see if it satisfies the restriction. Lastly, we finish our optimization on Zone A.

\subsubsection{Result}

We then make two figures considering the relation between waiting time, the optimized number of lanes and period of the day separately. We can come to a conclusion that optimizing the number of lanes in accordance to the time-varying model sufficiently reduces the probability of waiting in a queue. Moreover, according to Figure 5, variance of the $W_{p}$ with optimized lanes is dramatically reduced comparing to the average lanes. Now we want to estimate $\theta=E\left[W_{q 1}\right]$.
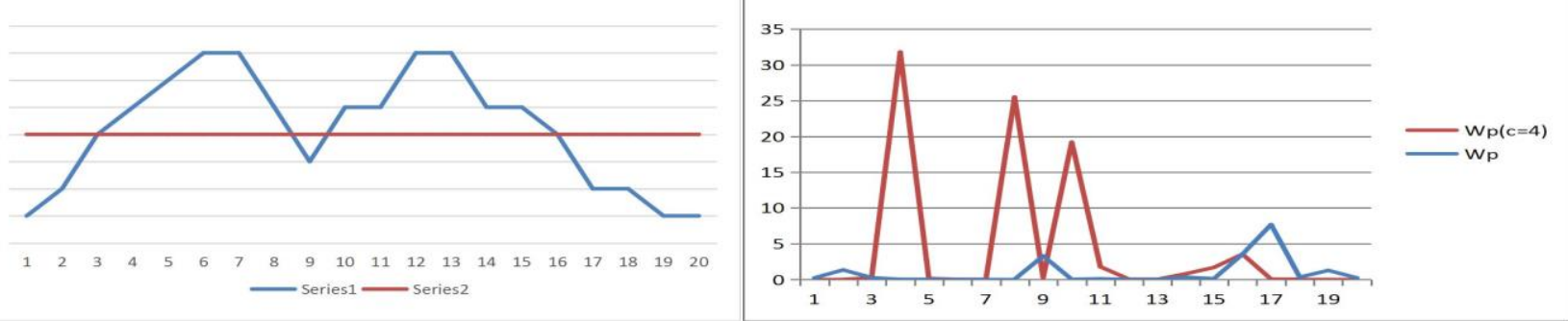

Figure 3: the best number and average number of lanes

Figure 4: the corresponding $W_{q}$

We know that $E\left[W_{q 2}\right]=\eta$, applying the control varieties method, we have the conclusion that for any constant $c$, the quantity 
$W_{q 1}+c\left(W_{q 2}-\tau\right)$

is also an unbiased estimator of $\theta$. Therefore,

$\operatorname{Var}\left(W_{q 1}+c\left(W_{q 2}-\tau\right)\right)=\operatorname{Var}\left(W_{q 1}+c\left(W_{q 2}\right)\right)$

The equation reaches its minimal value when $\mathrm{c}$ reaches its best value

$c^{*}=-\frac{\operatorname{Cov}\left(W_{q 1}, W_{q 2}\right)}{\operatorname{Var}\left(W_{q 2}\right)}$

and for this value the variance of the $\theta$ is

$$
\operatorname{Var}\left(W_{q 1}+c^{*}\left(W_{q 2}-\tau\right)\right)=\operatorname{Var}\left(W_{q 1}\right)-\frac{\left[\operatorname{Cov}\left(W_{q 1}, W_{q 2}\right)\right]^{2}}{\operatorname{Var}\left(W_{q 2}\right)}
$$

With the use of the equation, we reduce the variance up to 90 percent.

\section{Analysis on the Impact of Social Norms via Cellular Automaton Model}

In the previous sections, we did not take cultural differences into consideration. However, we can not neglect the fact that passengers in the flow may follow a specific pattern corresponding to the social norms. For example, Americans respect and prioritize the personal space of others, while Chinese prioritize individual efficiency. As a result, in an airport located in the U.S., the majority of the flow is more likely to keep a certain distance between two passengers, while in a Chinese airport, the queue are more likely to keep a fast pace. We thus analyze how the culture norms may impact our model.

\subsection{Cellular Automaton Model}

Wolfram denotes Cellular automaton as follows,

"A cellular automaton is a collection of 'colored' cells on a grid of specified shape that evolves through a number of discrete time steps according to a set of rules based on the states of neighboring cells. The rules are then applied iteratively for as many time steps as desired."

Comparing it with the passenger's behavior in queuing model, we can find there are many similarities:

-Each individual can be considered as a cell.

- Passengers in front and behind can be considered as neighboring cells.

- The reaction of an individual to the queue can be considered as the interaction rules among cells.

Therefore, we simulate the individual queuing behavior with the cellular automaton model.

\subsection{Assumptions}

To simplify the model, we make assumptions as follows.

-The size of each cell is $30 \mathrm{~cm} \times 30 \mathrm{~cm}$.

-The only input to the transition rules are the state of cells in the neighborhood. That is, no cutting line is allowed.

-There are two types of cells in the model (one lane), denoted as A and B, corresponding to passengers from two different cultures: Americans and Chinese.

-The ratio of the types is $1: 1$.

-Each type occupies a lane.

\subsection{Simulating Process in One Lane}

In this step, we divide each lane into 50 cells and define the interaction rules between neighborhood cells. First, we use a characteristic function to describe the current state $y=\left\{\begin{array}{l}1, \text { occcupied } \\ 0, \text { free }\end{array}\right.$

Then we analyze how Americans and Chinese differ in behavior.

-an American passenger tends to move when there are at least two grids in front of him/her is free.

- a Chinese passenger tends to move as soon as the grid in front of him/her is free. 
Further, we draw a graph defining the model.

We discuss the processes of the CA model: The speed $\mathrm{v}$ of each cell is $50 \mathrm{~cm} / \mathrm{s}$.

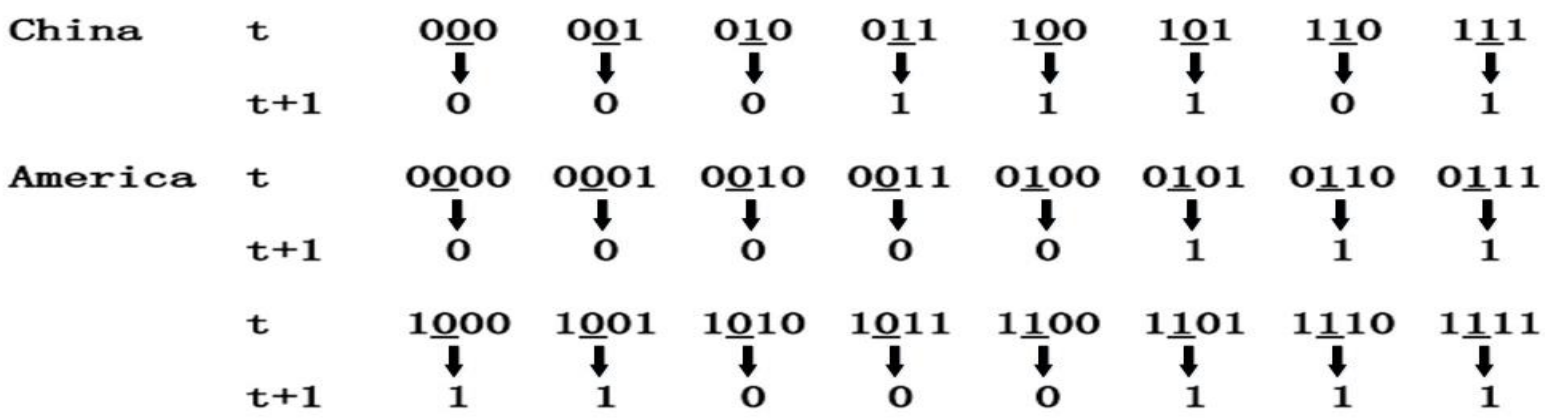

-Queuing process: Passengers wait to get checked in a queue.

-Moving process: Passengers move forward with the speed $\mathrm{v}$ when there are sufficient free grids ahead.

-Checking process: Passengers arrive at the checking point $\mathrm{i}$ and remain still for a period regarding to the checking rate.

-Leaving process: Passengers leaves the queue with the speed v.

There are three checking points where passengers are required to stay in our model: the ID checking section with $\mu_{1}=5: 308$, the millimeter scanner checking section with $\mu_{2}=5$ and the XRay checking section $\mu_{3}=15$. Apply the analysis above, we make the table below.

Table4: checking time in accordance to $\mu_{i}$

\begin{tabular}{llll}
\hline$\mu_{i}$ & American & Chinese & Mean \\
\hline 5.308 & 11.904 & 11.304 & 11.604 \\
15 & 4.6 & 4 & 4.3 \\
5 & 14.6 & 14 & 14.3 \\
\hline
\end{tabular}

\section{Conclusion and Discussions}

\subsection{Strengths}

- Integrity: We use the given data and simulates how the system works in actual cases.

- Flexibility: We take many factors into consideration as well as make adaptive assumptions, which is easy to make further assessment.

- Comprehensibility: We provide different visions to the model.

\subsection{Weaknesses}

-We neglect the individual difference in our model, which may cause flaws.

-We simplify the system and neglect the time cost in Zone C and Zone D.

-We assume that the direction of the flow is fixed. However, the direction may vary in real cases.

\subsection{Future Work}

We will continue working on our model until it fits the actual cases and solve all possible bottlenecks in the system. Here is how we are going to improve the model:

- collect more data for each section from different airports in different cultural backgrounds and make thorough analysis on stuffing.

- Apply combined assumptions into the model.

- Take individual differences into consideration and modify the current model.

\section{References}

[1] https://en.wikipedia.org/wiki/Jackson_network

[2] http://www.gbiac.net/en/lkfw/jess/security_channel 
[3] mathworld.wolfram.com/CellularAutomaton.html

[4] www.resultland.com

[5] Dorton,L,S, Analysis of Airport Security Screening Checkpoints using Queuing Networks and Discrete Event Simulation: A Theoretical and Empirical Approach, (2011).

[6] Liu,R.,Leone,K. The key design parameters of checked baggage security screening systems in airports. (2005).

[7] Wong, S.,Brooks, N. Evolving risk-based security: A review of current issues and emerging trends impacting security screening in the aviation industry. (2015)

[8] Ross,M.,S. Simulation,4th edition,London: Elsevier, (2006) 